\title{
e-Phaïstos
}

e-Phaïstos

Revue d'histoire des techniques / Journal of the history

of technology

I-1 | 2012

Le patrimoine maritime

\section{Les bateaux traditionnels de Bénarès, regards sur une technique de construction}

\section{Manuel Montanez}

\section{(apenEdition \\ Journals}

Édition électronique

URL : http://journals.openedition.org/ephaistos/244

DOI : 10.4000/ephaistos.244

ISSN : 2552-0741

Éditeur

IHMC - Institut d'histoire moderne et contemporaine (UMR 8066)

Édition imprimée

Date de publication : 1 juin 2012

Pagination : $39-49$

ISSN : 2262-7340

Référence électronique

Manuel Montanez, "Les bateaux traditionnels de Bénarès, regards sur une technique de construction », e-Phaïstos [En ligne], I-1 | 2012, mis en ligne le 01 janvier 2015, consulté le 08 mai 2019. URL : http:// journals.openedition.org/ephaistos/244 ; DOI : 10.4000/ephaistos.244 


\title{
Les bateaux traditionnels de Bénarès, regards sur une technique de construction
}

\author{
Manuel Montanez \\ doctorant au Centre d'Histoire des \\ Techniques (CH2ST/EA127), \\ Université Paris 1 Panthéon-Sorbonne
}

\section{Introduction}

La forme des bateaux traditionnels et la façon de les construire répondent à la logique et aux exigences de leur utilisation et de leur environnement. Elles sont en lien intime avec un milieu, comme le rapport existant entre une culture et son emprise géographique. Le bateau traditionnel est un objet technique endogène d'un paysage $^{1}$, un artefact «en subtile harmonie avec un milieu infiniment diversifié dont ils semblent émaner de façon presque naturelle» ${ }^{2}$, une réponse culturelle unique, en symbiose avec son environnement naturel et son espace socio-économique. Dans une culture mondialisée qui tend aujourd'hui à généraliser les habitudes et à mettre en danger de disparition des cultures locales, les bateaux traditionnels de Bénarès sont un exemple de patrimoine vivant et une facette de la mémoire liée à la nature d'un lieu. Cet article présente les bateaux en bois traditionnels de la ville de Bénarès, construits sur les berges du Gange : le rapport matériel entre forme, environnement et technique de construction. Puis il explore cette tradition dans des époques éloignées et aux alentours de Bénarès afin de trouver des références techniques et élargir le contexte où s'inscrit l'histoire du bateau de Bénarès.
Bénarès, sur les rives du Gange, a profité d'une position géographique privilégiée : au croisement de routes commerciales entre l'est et l'ouest du subcontinent et entre les vallées de l'Himalaya au nord, et du sud de l'Inde. Le Gange ayant longtemps été une route navigable a tenu une place essentielle dans l'économie et l'évolution de la ville ${ }^{3}$. Les bateaux ont joué un rôle important dans le transport du coton, de la soie, de l'opium et d'autres produits manufacturés de la ville. Parallèlement, la ville de Bénarès s'est développée en Asie du Sud comme un haut lieu de pèlerinage. Son paysage regorge de mythes et de légendes ${ }^{4}$ et le Gange joue un rôle essentiel dans les rites et les tâches quotidiennes des habitants et des pèlerins qui s'y rendent. Les bateaux accompagnent plusieurs de ces rituels et font partie du paysage sacré de la ville. Le fleuve, bénéfique pour plusieurs raisons, est aussi dévastateur : les bateaux affrontent périodiquement d'importantes variations de courant et de niveau d'eau du fleuve (plus de $15 \mathrm{~m}$ entre l'étiage et les crues) et sont adaptés à ces variations du milieu fluvial. Évoluant dans ce contexte culturel et géographique, les bateaux suivent le rythme du fleuve et participent des activités de la ville. Ainsi, on peut dire que, comme le Gange qui tient une place 
centrale dans la ville, les bateaux qui le sillonnent font intrinsèquement partie de son paysage.

\section{Le milieu fluvial de Bénarès : spécificités géo- morphologiques et culturelles d'une ville sa- crée}

La forme d'un bateau traditionnel dépend du site où il navigue et de la fonction pour laquelle il a été construit. Le milieu fluvial de Bénarès est un environnement géographique et hydrologique exigeant pour un bateau, à cause notamment des courants changeants, des vélocités fortes du fleuve et des rives de nature différentes. Aussi, les bateaux participent à une riche gamme d'activités fluviales et s'immergent dans un paysage sacré comme élément indispensable.

Bénarès, ou Vârânasî, se situe au nord de l'Inde, à $25^{\circ} 4$ '39" de latitude nord et $83^{\circ} 1^{\prime} 30$ "de longitude est, à mi-cours du Gange, sur un méandre du fleuve. Le fleuve ici change momentanément sa direction OuestEst pour celle Sud-Nord. La ville est construite sur la haute berge de la rive ouest, s'élevant à $20 \mathrm{~m}$ au dessus du niveau des crues du fleuve. Cette rive solide ${ }^{5}$ est recouverte de ghâts -ensemble de marches et de paliers qui descendent au fleuve. Construits en grès ${ }^{6}$, les ghâts s'adaptent à la topographie du site tout en la renforçant et la protégeant ${ }^{7}$. La rive est, à l'intérieur du méandre, constituée de dépôts sableux instables, est de faible hauteur ; libre de toute construction, elle s'inonde ou s'assèche selon les crues et les étiages du fleuve. La constitution de ces rives -ghât en pierre d'un côté et banc de sable de l'autre- influence, certainement, les formes et matériaux des coques de bateau.

La ville est bordée au Nord, au Sud et sur la rive ouest, par deux fleuves : respectivement la Varuna et l'Assi qui se déversent dans le Gange. À l'ouest de la haute berge, le terrain suit une pente douce qui au XIX ${ }^{\mathrm{e}}$ siècle culminait dans une série de lacs et d'étangs aujourd'hui asséchés. À cette époque, plusieurs descriptions et illustrations nous montrent une ville parsemée d'étendues d'eau ${ }^{8}$. La série de lacs et étangs communiquait et pouvait devenir un cours d'eau lors de fortes pluies. La ville, alors, était entourée d'eau et prenait l'aspect d'une île9. Le moine Fa-Hien en 399 av. J.C., mentionne « la ville entourée de rivières ${ }^{10}$. C'est dans cet environnement aquatique que les bateaux de Bénarès se sont développés.

Le climat de la région est subtropical-continental et comme dans toute l'Inde, les saisons de Bénarès sont rythmées par la mousson. Il est possible de parler de trois saisons : froide de novembre à février, chaude de mars à mi-juin et de pluies de mi-juin à octobre ${ }^{11}$. Le niveau de l'eau du Gange varie selon les saisons et atteint une hauteur maximum à la fin de la mousson avec l'eau des pluies torrentielles et la fonte des glaciers dans l'Himalaya. L'eau peut monter à Bénarès à plus de $15 \mathrm{~m}$ au dessus du niveau d'étiage et sa vélocité pendant la mousson être de $5 \mathrm{~m} / \mathrm{s}$, avec une décharge de $12,000,000$ cusecs $^{12}\left(34000 \mathrm{~m}^{3} / \mathrm{s}\right)$.

À Bénarès, le lit mineur ${ }^{13}$ se présente pendant la saison sèche, en hiver. La largeur du fleuve à cette époque se réduit entre $250 \mathrm{~m}$ et $500 \mathrm{~m}$ selon les endroits et sa profondeur est de 10m. Il y a aussi des crues exceptionnelles, alors l'eau peut monter jusqu'à 20m au dessus du niveau d'étiage inondant certaines zones de la ville ${ }^{14}$. Dans son sens longitudinal il y a sûrement des accidents au fond du fleuve qui nous sont inconnus. Ils permettraient de parler de fonds maigres ou de fonds mouilles pour ainsi identifier des zones avec différents degrés de navigabilité ${ }^{15}$. Plusieurs entretiens oraux réalisés auprès de bateliers nous ont informés sur les courants du fleuve. De manière générale, le courant est plus important au milieu du fleuve. Durant la mousson, il augmente près des rives. La navigation des bateaux vers l'amont devient donc extrêmement ardue durant la saison des pluies. Au moins trois bateliers sont nécessaires pour faire avancer un petit bateau qui d'ordinaire n'aurait besoin que d'un seul rameur.

Les ghâts qui s'avancent vers le fleuve sont ordinairement les plus frappés par les courants. En revanche, ceux en retrait ont des eaux plus calmes. Il existe aussi des contre-courants : Rai Anand Krishna nous parle d'un contre-courant sur la rive droite qui expliquerait la présence de bateaux de ce côté-la, avançant vers l'amont ${ }^{16}$. Mais cette affirmation est contredite par le 
batelier Ajit Sahani : "Non, il n'y a pas de contre-courant sur la rive opposée. Il y a, par contre, beaucoup de vents et de vagues dans le sens opposé au courant $»^{17}$. Lors de notre entretien avec le batelier Deepu, nous avons aperçu un contre-courant qui existe aussi entre Panchganga et Bhonsle ghât. On l'appelle le Tribeni Ganga (ou le Gange du confluent) et sa présence augmente la sacralité du lieu. Un bateau, à cet endroit, remonte le fleuve sans besoin d'être propulsé. Plusieurs tourbillons sont présents sur le fleuve, surtout durant la mousson. Ceux qui se trouvent près des rives sont les plus dangereux pour les bateliers.

Résumer la culture qui s'est matérialisée dans ce paysage n'est pas l'objet de la présente étude, aussi, nous renvoyons aux différents ouvrages signalés dans la bibliographie. Cependant, placer le bateau et sa technique constructive dans son contexte culturel impose d'évoquer sa participation dans les deux plus importants rituels qui se développent sur la berge : les pèlerinages et les rites de crémation. Des milliers de pèlerins viennent chaque année dans la ville et se déplacent souvent par bateau pour visiter les nombreux temples de la ville. Ce moyen de transport est plus pratique et confortable. Selon la taille des groupes de pèlerins, des bateaux de tailles différentes organisent des parcours vers les ghâts et les temples les plus imortants. Aussi, la ville de Bénarès est considérée comme un tirth ou lieu de passage d'une rive à l'autre et, plus symboliquement entre la vie et la mort. C'est pourquoi, beaucoup de dévots hindous sont incinérés sur les rives de Bénarès afin de les libérer du cycle des réincarnations. Au terme de ce rituel, le bateau joue un rôle important en transportant les cendres du défunt au milieu du Gange pour y être jetées.

\section{Les bateaux actuels et leur description}

Les bateaux sont amarrés tout près de l'endroit où habite le batelier, les éléments architecturaux du ghât sont utilisés pour s'amarrer. Quand il n'y a rien de visible, de gros clous sont enfoncés dans les joints des pierres comme ancrage. De la même manière « N'importe qui peut venir construire son bateau sur les ghâts. Souvent on le fait là où nous habitons. Il faut juste ne pas déranger les pèlerins... pas être trop près de l'eau où ils font leurs ablutions $»^{18}$. Les bateaux, bateliers et Mistri (maitres charpentiers), sont constamment présents sur les rives. C'est sur ces rives qu'un travail de terrain a été réalisé en 2009, des relevés des bateaux ont été réalisés ainsi qu'une série d'entretiens avec les acteurs participant à la matérialisation et à l'utilisation des bateaux.

Grâce à cette étude nous avons pu différencier par leur morphologie quatre types de bateaux : Doonghy, Bajra, Ghâtaha et Kattar. Nous avons relevé la forme de chaque type de bateau, les différentes parties qui le constituent ainsi que ses matériaux de construction. Les trois premiers de ces quatre types de bateaux ont le même principe architectural (voir glossaire) et utilisent le bois comme matériau pour leur bordé. Tous trois ont des noms d'origine hindi. Le quatrième type de bateau, le kattar, est construit à charpente intérieur avec une armature en bois et un bordé en tôle. Son fond est plat, légèrement cintré et constitué d'une sole en bois (voir glossaire). Le nom de ce bateau a probablement pour origine l'anglais cutter, qui désignait une petite embarcation d'un faible tirant d'eau permettant d'amener les passagers des grands cargos vers la rive. C'est le bateau le moins cher et le plus simple à construire, mais c'est aussi celui qui présente le plus de dangers, de problèmes et d'accidents, preuve qu'il n'est pas totalement adapté au lieu. Nous nous concentrerons ici sur les trois premiers types de bateaux, décrits au travers du mode de construction de la coque. Ces trois types de bateaux sont construits en bois. Les bateaux sont objet de fierté pour les bateliers de Bénarès, selon un batelier, leurs bateaux sont

"Mieux que ceux d'Allahabad ou de Haridwar et Ri-
shikesh ${ }^{19}$. Ces derniers sont enveloppés de tôles alors
que les nôtres sont entièrement en bois et durent
donc plus longtemps. Leurs bateaux sont conçus
pour des rivages en terre. Ici, à Bénarès, les berges
sont en pierre massive ; les bateaux doivent se pro-
téger et résister aux impacts contre la pierre ${ }^{20}$.

Nous allons rapidement décrire ces trois types de bateaux possédant une même technique de construc- 


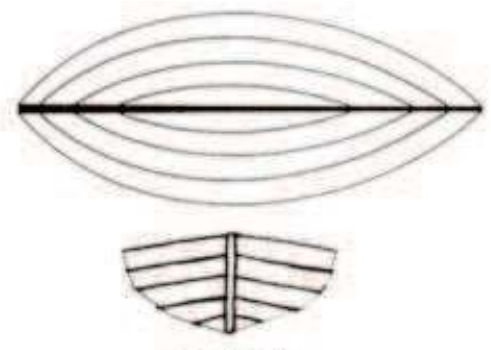

a borde premier
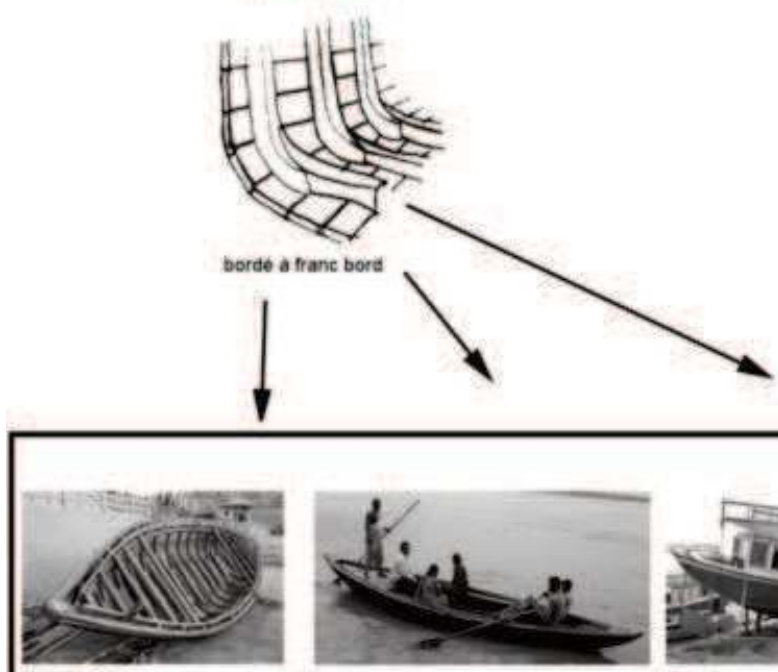

GHATAHA

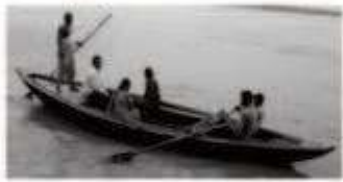

DOONGHY
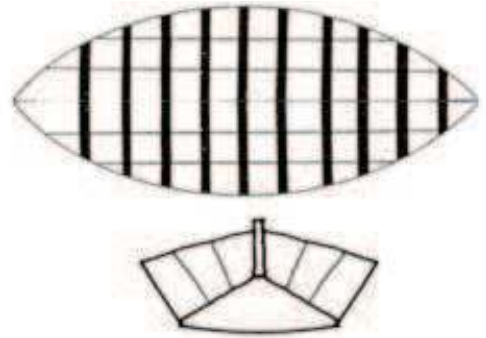

Fig. 1 : Principes architecturaux des coques des bateaux traditionnels de Bénarès (M. MONTANEZ)

tion. Seul l'usage auquel ils sont destinés incite à des différences de forme remarquables.

\section{Doonghy}

Le doonghy est le bateau banarasi par excellence. Son profil est facilement reconnu et les bateliers parlent de ses belles formes avec une certaine fierté. Seuls les Mistris, charpentiers locaux sont, selon eux, habilités à les construire. C'est le type de bateau le plus répandu à Bénarès ; il est conçu pour transporter jusqu'à 15 passagers et ses dimensions peuvent varier de $5 \mathrm{~m}$ à $12 \mathrm{~m}$ de longueur par 1,5m à $3 \mathrm{~m}$ de largueur. Le francbord varie de 0,5 à 1,5m et le tirant d'eau de 10 à $30 \mathrm{~cm}$. conatrute sur sola

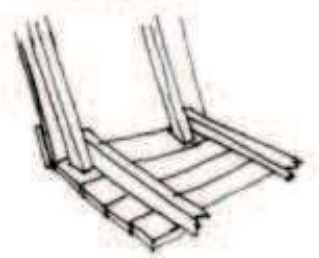

1

Son fond est semi plat avec une tonture creuse peu prononcée et avec un avant et un arrière pointus. L'arrière est plus élevé que l'avant. Il présente un pontage complet et plat qui entoure une grande écoutille centrale, de forme carrée, rehaussée d'une hiloire. Quelques panneaux sur le pont, au niveau de la première tierce à l'avant, peuvent être retirés afin de découvrir le banc de nage pour un ou deux rameurs.

Son fond semi plat est un peu cintré ; il est structuré autour d'un axe constitué par une virure de quille qui intègre l'étambot et l'étrave. L'étambot et l'étrave sont les deux pièces clés de toute la structure ; ils sont taillés en bois massif et conçus spécialement pour recevoir les bordés. Le premier bordé, le galbord, est collé à la quille alors que les trois dernières virures supérieures son incrustées ensembles à l'étrave et l'étambot dans la râblure.

Des varangues et des allonges sont posées pour renforcer la forme de la coque. Des allonges sont, à leurs arrivées au plat-bord, entourées par une préceinte à l'extérieur, et à l'intérieur par une serre bauquière. Il y a une continuation des allonges pour accouder les toletières à l'avant au niveau du banc de nage. Les quatre bittes aussi, deux à l'avant et deux à l'arrière sont 
accoudées aux membrures. On trouve trois renforts longitudinaux intégrant les membrures : une serre de banc, une serre bauquière et deux serres de pavois (une interne et une externe couronnent le bordé, une sorte de préceinte doublée). La coque est revêtue d'une couche d'alkatara (goudron) à l'extérieur comme à l'intérieur.

Pendant la saison sèche, lorsque l'eau est basse et que le courant est faible, un seul rameur suffit pour conduire le bateau. Pendant la mousson, quand la force et la vitesse du courant augmentent, l'équipage est composé de deux rameurs à l'avant et un rameur à l'arrière qui dirige l'aviron de gouverne (khilwari). On peut utiliser les deux avirons, un de chaque côté du bateau, comme des mâts, pour dresser une voile sommaire.

\section{House-boat ou Bajra}

Bateau avec une superstructure fixe, permettant le transport et le séjour de passagers avec commodité. Totalement construit en bois dans sa version originale ce bateau est conçu pour transporter 50 à 120 passagers. On trouve des house-boats de taille différente, 8 à $18 \mathrm{~m}$ de longueur par 2,5 à $5 \mathrm{~m}$ de largeur, avec un tirant d'eau de 30 à $70 \mathrm{~cm}$. L'arrière est beaucoup plus élevé et pointu que dans le doonghy et l'avant plus arrondi. Il est plus large et son fond beaucoup plus plat que le ghâtaha ; un ghâtaha peut être transformé en houseboat (c'est le cas du bateau que nous avons relevé).

La superstructure localisée au centre et à l'arrière du bateau recouvre deux tiers de sa longueur et la totalité de sa largeur. Elle héberge une salle entourée par des baies avec des persiennes. Un plancher posé sur les allonges de la coque unifie le sol de la salle. Deux bancs courent de chaque côté de la salle en dessous des baies et s'appuient sur les membrures de la coque. Le centre est dégagé ; quelques poutres seulement coupent l'espace pour soutenir le rouf plat du salon qui est aussi utilisé comme plateforme extérieure pour les passagers.

Le bateau est ponté à l'arrière et à l'avant, en dehors de la superstructure. On trouve deux portes sur la fa- çade avant du salon, une de chaque côté de l'axe longitudinal. L'accès au salon se fait par la porte à droite ; on y trouve quelques marches pour descendre au niveau du plancher de la salle. La porte à gauche est une porte fenêtre devant laquelle une échelle permet de monter au rouf. Au fond de la salle, une autre porte permet d'accéder par deux marches au pont arrière où se trouve la barre du gouvernail. Quelques house-boats ont des W.C. au fond de la salle.

À l'extérieur, le long du plat-bord de la coque, un pavois courre et passe sous les baies de la salle pour se prolonger ensuite sur les deux ponts. Deux bittes marquent sa fin à l'avant et à l'arrière et marquent les accès du bateau. À l'avant de plusieurs house-boats, on peut aussi trouver des toletières qui témoignent d'un passé où le house-boat était un bateau à aviron. À l'extérieur et entourant la superstructure, on trouve une série de bâtons attachés au garde-corps du rouf et au plat-bord de la coque tout au long de la hauteur de la salle ; ce sont des protections ou des défenses pour amortir les chocs ou empêcher le frottement ou racage contre d'autres navires.

La plupart des house-boats sont motorisés depuis 10 ans ; un moteur de 10 à $18 \mathrm{HP}$ se trouve à l'intérieur de la salle, juste avant de sortir sur le pont arrière, dans une grosse boite en tôle. L'arbre de l'hélice se trouve sous le plancher ; l'arrière étant surélevé, la plus large partie de l'arbre se trouve à l'extérieur soutenu par un bras métallique accroché au bordé. La coque est percée pour laisser passer l'arbre de l'hélice. Cette modification est réalisée avec une grande économie de moyens. Le réservoir d'essence se trouve sous le pont arrière. L'hélice est fabriquée artisanalement dans la ville.

À l'arrière sur le pont, une jaumière est percée sans toucher l'étambot pour laisser passer la mèche du gouvernail. Un gouvernail rectangulaire et métallique a remplacé le beau gouvernail triangulaire en bois traditionnel. Il est soutenu par le dernier barrot à l'arrière qui est renforcé. Une large barre de gouvernail est dirigée par une personne qui peut être debout sur le pont en regardant au dessus du rouf ou bien assise sur le rouf même. Une perche en bambou est utilisée pour 
manœuvrer le départ et l'arrivée aux ghâts. Une place est réservée à l'avant de la superstructure pour installer un mât ; on voit en effet des accroches entre les deux portes, le pont est percé à cet endroit et au fond de la coque on trouve un parage sur une allonge pour recevoir le mât.

Le bordé a un doublage en tôle pour le renforcer. L'intérieur de la coque et les ouvres vives à l'extérieur sont recouverts d'une couche d'alkatara. L'étrave et l'étambot sont quelque fois taillés ou peints pour représenter des têtes d'homme ou d'animal.

\section{Ghâtaha}

C'est un bateau conçu avec une grande capacité de charge pour le transport de gros poids et éventuellement d'un grand nombre de passagers. Avec des formes similaires au doonghy, on le différencie de celui-ci surtout par sa taille : de 12 à $25 \mathrm{~m}$ de longueur et de 3 à $5 \mathrm{~m}$ de largeur. Son arrière est plus élevé et son franc bord et tirant d'eau beaucoup plus grands. Son principe architectural est le bordé actif. Il est construit suivant la même procédure que le doonghy, bien que les dimensions soient supérieures à celui-ci : étrave et étambot unis par une virure de quille ; un galbord plus large assemblé à la quille et aux côtés inférieurs de l'étambot et de l'étrave ; le galbord avec le ribord et trois ou quatre virures en plus forment le fond de la coque.

Une râblure dans l'étambot et l'étrave reçoit les trois dernières virures qui font la forme avant et arrière du bateau et couronnent le premier groupe qui font le bordé de fond. Le nombre de virures peut varier pour élargir le franc-bord. Il y a plus d'allonges, de varangues et de renforts transversaux que dans le doonghy pour lui donner plus de résistance. Les plats-bords sont formés par une préceinte à l'extérieure et par une serre bauquière à l'intérieur ; elles forment une fargue qui coure le long du bord, de l'étrave à l'étambot.

Les jambettes de fargue reçoivent également deux à quatre toletières pour les avirons et quatre bittes pour les amarres. Dans quelques ghâtaha une ou deux virures sont ajoutées sur le plat-bord pour augmenter le franc-bord. Elle surpasse aussi l'étrave et l'étambot et fait une sorte de muraille continue. À l'arrière du bateau, comme dans le house-boat, une jaumière est percée sans toucher l'étambot, pour laisser passer la mèche du gouvernail métallique. Un gouvernail rectangulaire et métallique remplace presque toujours le gouvernail triangulaire en bois traditionnel attaché à l'arrière tribord. Sa grande hauteur à l'arrière permet un meilleur contrôle physique sur le bateau : un gouvernail avec un long bras de levier et un bon contrôle visuel sur la marche du bateau. Les bateaux très lourds qui ne peuvent pas être retirés de l'eau chaque année pour être calfeutrés d'alkatara ont la coque recouverte de tôle.

Les grands ghâtaha, spécialement conçus pour le transport de grandes quantités de sable et de bois sont appelés mahalya. Après l'interdiction d'exploitation de sable il y a une quinzaine d'année les mahalya ont souffert d'une réduction de leur franc-bord (élimination de quelques virures) et ont été pontés pour transporter des passagers. Souvent ces bateaux de passagers sont ornés d'une superstructure légère et éphémère qui permet de faire un peu d'ombre aux passagers ; on les trouve aussi embellis de fleurs et de guirlandes.
«On utilisait de très gros bateaux pour emporter le sable, appelés Mahaliya ${ }^{22}$. Ces grands bateaux ont été réduits en taille pour devenir des bateaux pour passagers. Plusieurs lisses supérieures ont été enle- vées. Les voiles aussi ont été remplacées par des mo- teurs. Les seuls mahaliyas qui restent aujourd'hui sont utilisés dans le transport de bois (de crémation) à Manikarnika ghât»"22.

Un ghâtaha peut aussi être appelé dihati. Les dihati sont conçus à l'origine pour porter de la marchandise. Ils ne sont pas pontés. Ils sont moins finis mais plus robustes. Ils portent sable, bois et autres marchandises lourdes qui les submergent des fois jusqu'au niveau des plats-bords. Le terme Dihat signale des endroits qui se trouvent à l'extérieur de Bénarès ${ }^{23}$, où sont construits ces bateaux, d'où leurs noms ${ }^{24}$.

\section{Une approche de la technique constructive de la coque}

La phase de conception du projet démarre avec la 
commande et la projection du bateau, le projet est ajusté au travers d'accords oraux entre le commanditaire et le Mistri, héritier d'un savoir-faire transmis de génération en génération par le geste et la parole. Il n'y a pas d'élaboration de plans ; le commanditaire fournit les matériaux nécessaires pour la construction et se charge des dépenses du chantier qui est monté sur le ghât le plus proche de sa maison.

La phase de construction démarre avec l'élaboration des deux pièces clés de la structure : l'étambot et l'étrave. Ces deux éléments unis par une virure de quille (Danr) forment l'axe à partir duquel le bordé se développe. Les bordages sont d'abord étuvés ou mis au feu pour les cintrer, puis, ils sont assemblés par des agrafes ou crampons métalliques disposés tout les $20 \mathrm{~cm}$ ap- proximativement, enfin, ils sont enfoncés dans des gorges entaillées dans les bords inférieurs internes de chaque bordage. Les coutures sont calfatées avec un cordon saturé de goudron.

Plusieurs kutta (ou chien, de l'instrument qui ressemble à une gueule de chien) permettent de courber la planche pour l'asseoir parfaitement au bordé en construction. Des allonges et des membrures sont posées pour renforcer la forme de la coque. On trouve trois renforts longitudinaux intégrant les membrures et servant de banc, de bauquière et de virures de pavois (une interne et une externe couronnent le bordé, une sorte de préceinte doublée). La coque est revêtue d'une couche d'alkatara (goudron) à l'extérieur comme à l'intérieur.
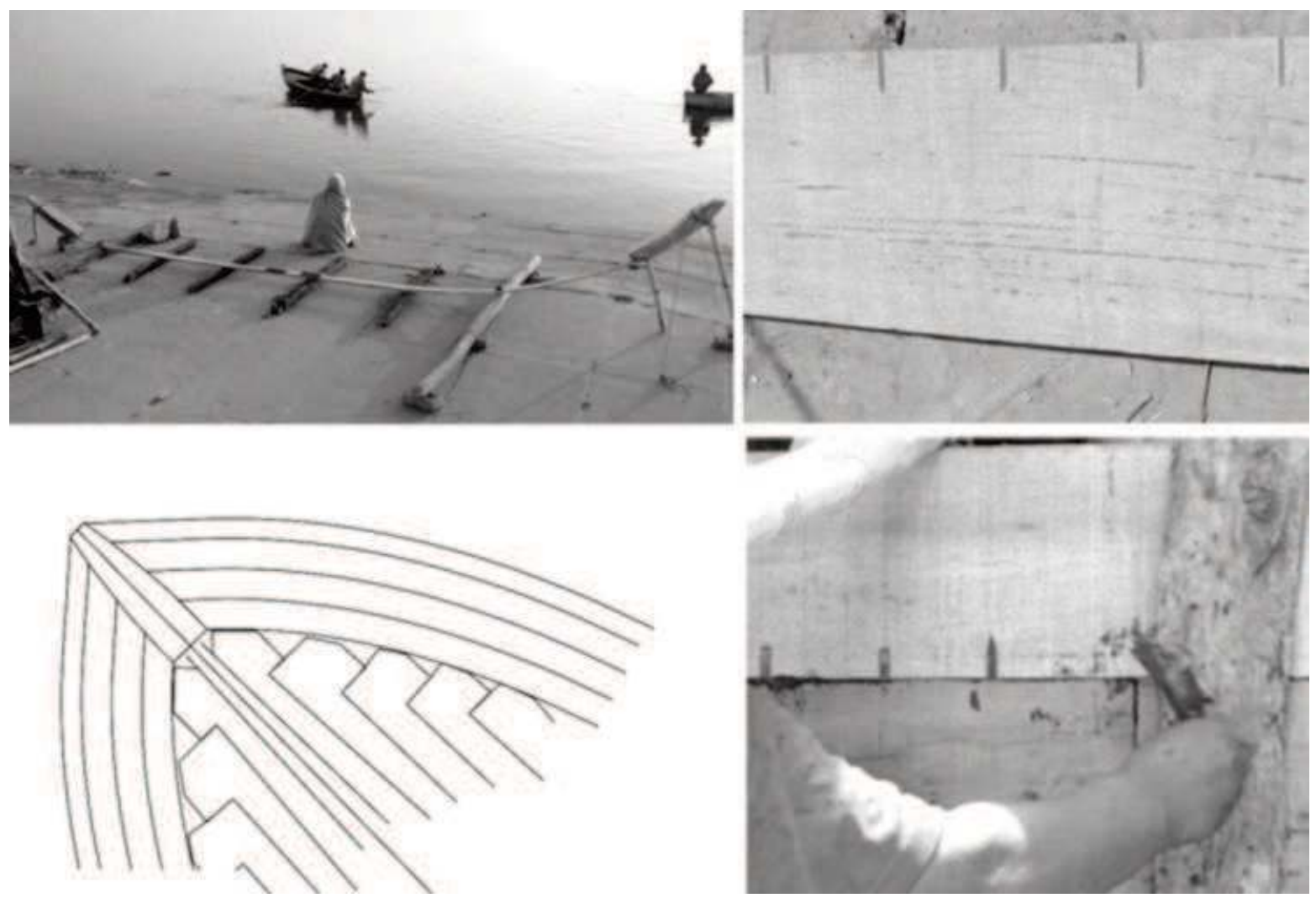

Fig. 2 : Démarrage de la phase de construction des coques, schéma de l'organisation des virures et photos de l'agrafage des bordés (M. MONTANEZ) 
Recherche historique d'une technique, des limites chronologiques et géographiques élargies

À partir de la technique constructive, ont été recherchés des rapports entre les bateaux de Bénarès et ceux qui ont existé aux différentes époques et en divers lieux sur le Gange. Leur technique de construction permet de restituer une partie de l'histoire des bateaux de Bénarès. Les témoignages récoltés et les observations réalisées dans ce travail de terrain se réfèrent logiquement à un passé récent. Je les ai confrontés à des données issues de différents types de sources :

-Il n'y a pas de sources archéologiques ; des fouilles archéologiques faites au nord de la ville dans les années 1960 n'ont révélé aucun objet en bois, ou en métal pouvant appartenir à l'architecture navale.

-Il n'existe pas de dessins techniques élaborés par les constructeurs, nous avons vu que le projet du bateau passe de la commande à la matérialisation sans passer par les plans.

-L'apprentissage du charpentier Mistri se fait par le geste et la parole et ne laisse aucun document graphique.

Constater la permanence de cette technique de construction dans la mémoire de la ville nous a amené à plonger dans les archives historiques - images populaires et scientifiques - afin d'identifier sa présence au cours des trois derniers siècles. Les peintures, gravures et photos retrouvés, décrivent les activités sur le bord du Gange et représentent des bateaux participant à la vie du fleuve. Les peintures et gravures anciennes faites par des Occidentaux sur les différents aspects de Bénarès donnent souvent un aperçu des bateaux présents dans la ville aux XVIII ${ }^{\mathrm{e}}$ et XIX ${ }^{\mathrm{e}}$ siècles et quelques panoramas du front de berge dessinés par des artistes locaux, témoignent aussi d'une importante activité nautique sur le fleuve. Alors que ces images permettent d'encadrer les bateaux et les batelleries participant du paysage, ils ne documentent pas directement les détails techniques de construction.

La recherche iconographique a été élargie jusqu'à embrasser les bateaux très variés de la baie du Bengale où se déverse le Gange, qui ont en outre été étudiés et répertoriés à partir du XVII ${ }^{\mathrm{e}}$ siècle par des voyageurs européens ${ }^{25}$. Ainsi, les témoignages de François Balthazar Solvyns et de l'Amiral François-Edmond Pâris deviennent indispensables pour notre recherche, ils attestent d'une constante : l'utilisation d'agrafes pour lier les bordages dans les bateaux du bassin du Gange (Fig. 3a et b).

François Balthazar Solvyns (1760-1824), est un artiste et voyageur flamand qui a parcouru la vallée du Gange entre 1791 et 1803 . Il dessine,comme on peut voir dans la Fig 3a, et écrit cette description d'un dinguy:

«Voici une autre espèce de barque, nommée dinguy, et servant à transporter les briques. L'estampe représente cette barque à terre : j'ai choisi cette position, afin de pouvoir montrer la construction des barques hindoues en général. Pour construire un bateau, les hindous commencent par choisir un grand morceau de bois, qu'ils couvrent à volonté. Aux deux extrémités de cette pièce ils attachent une autre pièce plus épaisse que la principale. Ils couvrent ensuite de planches cette simple charpente : mais ils ont une manière particulière d'attacher les planches les unes aux autres ; c'est par le moyen de crampons plats à deux pointes, qui entrent dans les planches qu'ils veulent lier : ils ne font usage des clous ordinaires que pour attacher les bords aux varangues. Pour former le bord du bateau on emploie des pièces de bois qui dépassent les planches. Cette manière est aussi simple que solide. Ce procédé de lier les planches mériterait d'être imité en Europe. ${ }^{26}$

L'Amiral François-Edmond Pâris prend des notes et relevés dans la première moitié du XIX ${ }^{\mathrm{e}}$ dans la baie du Bengale, ils sont publiés dans son Essai sur la construction navale des peuples extra-européens ${ }^{27}$, œuvre fondatrice de l'ethnographie navale. Là, en parlant des bateaux du Bengale, il offre la vision d'une technique constructive proche de celle utilisée à Bénarès actuellement :

« ... ses bordages sont réunis, en outre, par une quantité de petites crampes de fer disposées a l'intérieur et à l'extérieur comme le montrent les fig. 6 et 7 (pl. 32) [figure $3 \mathrm{~b}$ ]. Ces crampes c c, souvent en- 


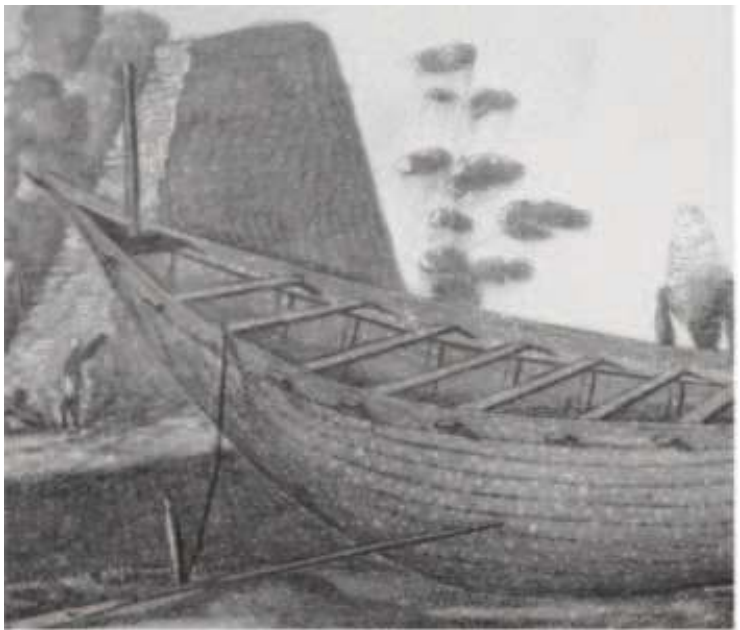

a.

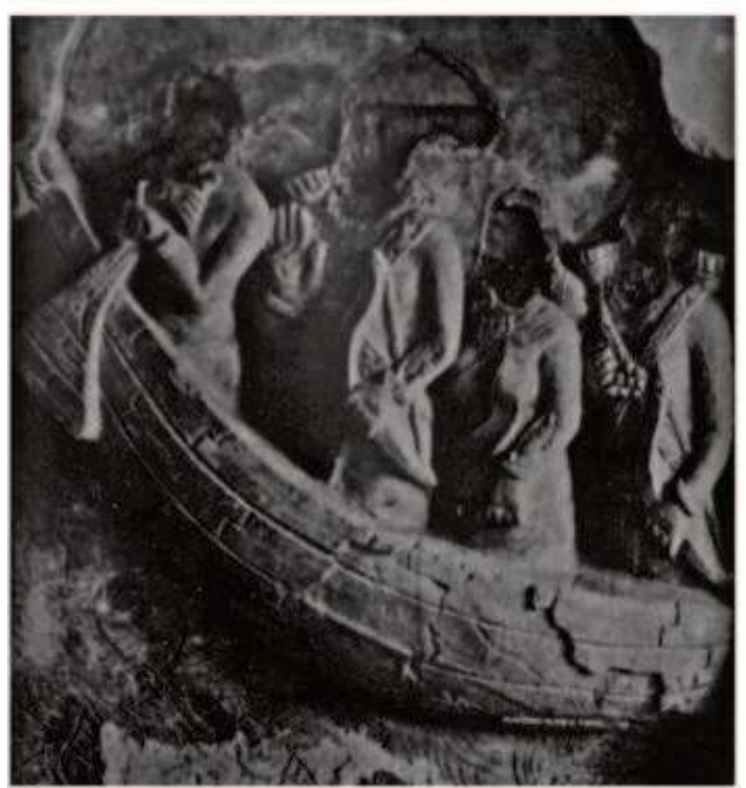

c.

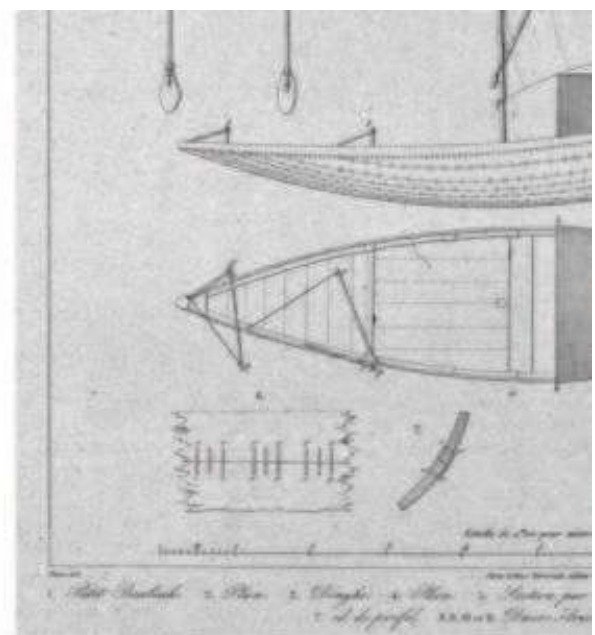

b.

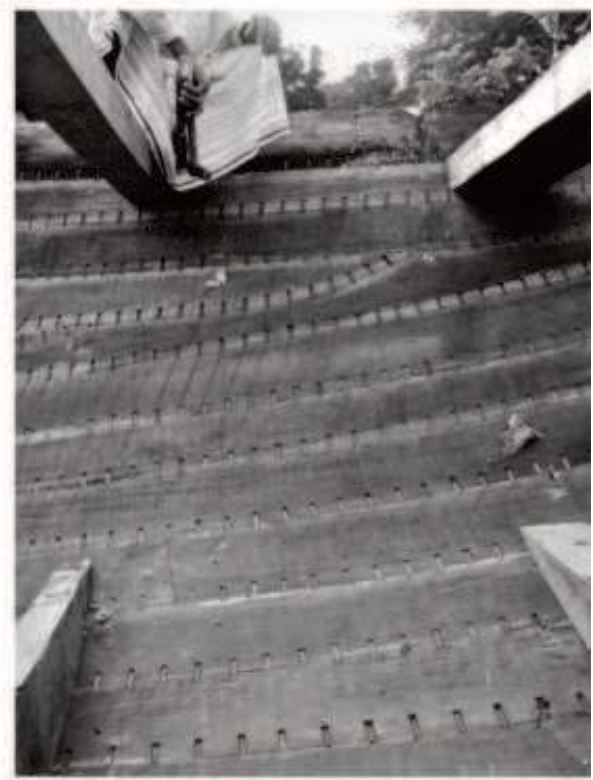

d.

Fig 3 : Images de coques à franc-bord du bassin du Gange :

« dinguy» par F. B. Solvyns

« dinghi » par l'Amiral Pâris

Bateau du Gange, relief sculpté (période Gupta)

Vue de l'intérieur de la coque d'un bateau traditionnel du golfe du Bengale en 2005

(Y. MARRE). 
foncées dans une rainure et rarement recouvertes de mastic, durent, dit-on, très longtemps, quoi que exposées au contact continuel de l'air et de l'eau ; elles lient si bien les bordages, que la couche de coton placée entre eux suffit pour s'opposer à toute infiltration. La forme oblique de leurs pointes est en partie, la cause de leur tendance à serrer, et leur grand nombre doit beaucoup accroître cet effet : l'usage en est très répandu au Bengale, où on les adapte à toutes sortes de bateaux, en les disposant par groupes de deux, trois ou quatre. $»^{28}$

Des recherches iconographiques autour des bateaux traditionnels, dans l'art indien de régions proches -bassin du Gange et golfe du Bengale- et dans le souscontinent indien en général ont mis en évidence une des premières représentations du bateau fluvial en Inde. Il s'agit d'un relief sculpté, bien conservé, sur un socle datant de la première moitié du VII ${ }^{\mathrm{e}}$ siècle ${ }^{29}$ (période Gupta), représentant un bateau avec un arrière surélevé et des bordages unis à franc-bord parfaitement visibles. Un des personnages dans le bateau dirige un gouvernail triangulaire. Cette image pourrait étendre les limites chronologiques de la présence de ce type de bateau dans la région, qui devrait être confirmée par des nouvelles données et des sources encore à trouver (Fig. 3c).

\section{Conclusion}

Historiquement, la technique de construction des bordés agrafés à franc-bord s'avère liée à une région particulière : le bassin du Gange, son delta et la baie où il se déverse. Elle est encore vivante aujourd'hui ${ }^{30}$ (Fig. 3d) et bien que les limites temporaires de sa présence soient encore à confirmer, son utilisation est attestée dans les constructions de bateaux dès la fin du XVIII ${ }^{\mathrm{e}}$ siècle. L'élargissement des limites géographiques et chronologiques de la recherche permettra, peut-être, de reconstituer la genèse du bateau traditionnel de Bénarès. Enfin, le rapprochement de cette technique avec celles utilisées dans des régions voisines inscrira ses formes dans l'arbre généalogique des bateaux de l'Asie du Sud.
1 On utilisera ce terme dans son acceptation géographique la plus courante, à savoir espace dont la physionomie et les dynamiques contemporaines résultent principalement de processus biophysiques mais qui sont, à des degrés divers, façonnés par les actions anthropiques.

2 BEAUDOUIN François, Bateaux des fleuves de France, Douarnenez, L'Estran, 1985, p. 234.

3 KOSAMBI Damodar Dharmanand, The culture and civilization of ancient India in Historical outline, New Delhi, Vikas, 1970.

4 Parmi de nombreux écrits voir ECK Diana L., Banaras : City of light, New York, Alfred A. Knopf, 1993 (1982 1ère edition) ; GUTSCHOW Niels, Benares : The sacred Landscape of Varanasi, London/Stuttgart, Axel Menges, 2006.

5 Cette haute berge est constituée d'un mélange d'alluvions et de kankar (dépôt calcaire), voir SINGH Reginald Lal, Banaras : A study in Urban Geography, Banaras Hindu University, Varanasi, Nand Kishore and Bros., 1955, p. 18.

6 Extrait de carrières qui se trouvent $20 \mathrm{~km}$ en amont du fleuve.

7 JALAIS Savitri, Protéger la rive, protéger la ville : le rôle structurel des ghât de Bénarès : 23èmes Journées Scientifiques de l'Environnement - Risques environnementaux : détecter, comprendre, s'adapter, Créteil, France, 2012.

8 Voir plan de 1828 dans PRINSEP James, Benares Illustrated, London, 1831.

9 ECK, op. cit., 1993, (1ère édition1982), p. 212.

10 Récits du pèlerin bouddhiste, Fa-hien, au IIIe siècle av. J.C.

11 SINGH, op. cit., 1955.

12 JOSHI E. B., Uttar Pradesh District gazetteers, Varanasi, 1965 (1st edition 1928).

13 Le lit mineur ou lit d'étiage est le niveau le plus bas du fleuve qui, même lors des plus basses eaux, n'est jamais asséché. Le lit majeur est le niveau d'eau maximal sans dépasser le niveau saisonnier habituel. Un champ d'inondation est un lit majeur exceptionnel lorsque les zones sont inondées durant les crues prononcées.

14 SINGH, op. cit., p. 21.

15 RIETH Eric, Des bateaux et des fleuves : Archéologie de la batellerie du néolithique aux temps modernes en France, Collection des Hespérides, Paris, Errance, 1998, p. 20.

16 KRISHNA Rai Anand, Banaras in the early $19^{\text {th }}$ century, Riverfront panorama, Varanasi, Indica, 2003, p. 8.

17 Entretien avec Ajit Sahani alias Babou.

18 Entretien avec le batelier Madan Majhi à Man Mandir Ghat.

19 Villes situées plus en amont sur le Gange.

20 Entretien avec Madan Majhi, batelier à Man Mandir Ghat.

21 Maha veut dire grand.

22 Entretien avec le batelier Madan majhi à Man Mandir Ghat.

23 Les villages qui entourent les petites villes sont généralement appelés dihat.

24 Entretien avec le batellier Deepu à Munshi Ghat. 
25 BOWREY Thomas A., Geographical Account of the Countries round the Bay of Bengal, London, R. Temple, 1905, p. 228 et 277. On trouve les premiers dessins réalisés et publiés en Europe en 1670 de cinq bateaux de l'Est de l'Inde (patela, ulák, bajarâ, purgo et bhar).

6SOLVYNS François Balthazar, Les Hindous, Paris, F. B. Solvyns et H. Nicolle, 1808-1812, Tome 3. Pl. 26

27 PÂRIS François-Edmond, Essai sur la construction navale des peuples extra-européens, Paris, A. Bertrand, 1841,

28 PÂRIS François-Edmond, Essai sur la construction navale des peuples extra-européens, Paris, A. Bertrand, 1841, p. 42. Ce texte est accompagné des premiers plans techniques des bateaux traditionnels de la région.

29 Ce bas relief a été découvert dans un temple dédié a Vishnu dans les années 1970 au Bihar dans la vallée du Ganges à 300km de Bénarès. Voir ASHER Frederick M., The art of Eastern India, 30o-80o, Minneapolis, The University of Minnesota press, 1980.

30 Swarup Bhattacharya amène une étude autour des charpentiers nautiques de Balagarh en West Bengal : http://www.projects.khojkolkata.org/boatpr.html (mars 2012)

\section{Bibliographie :}

COUTE Pierre-Daniel, LEGER Jean-Michel, Bénarès : Un voyage d'architecture / An architectural voyage, Paris, Créaphis, 1989.

GAENSZLE Martin, GENGNAGEL Jörg, Visualizing Space in Benares : Images, Maps and the practice of representation, Wiesbaden, Harrassowitz Verlag, 2006.

HAVELL Ernest Binfield, Benares, the Sacred City, London, Blackie and son, 1905.

JALAIS Savitri, Protéger la rive, protéger la ville : le rôle structurel des ghât de Bénarès : 23èmes Journées Scientifiques de l'Environnement - Risques environnementaux : détecter, comprendre, s'adapter, Créteil : France (2012)

PÂRIS François-Edmond, Essai sur la construction navale des peuples extra-européens, Paris, A. Bertrand, 1841

RIETH Éric, Archéologie de la batellerie et architecture nautique fluviale, Conflans Sainte-Honorine, Association des Amis du Musée de la Batellerie, 2006.

SHERRING Matthew Atmore, The sacred city of the hindus : An account of Benares in ancient and modern times, London, Trubner and co., 1868.

SOLVYNS François Balthazar, Les Hindous, Paris, F. B. Solvyns et H. Nicolle, 1808-1812. 